J Neurol Neurosurg Psychiatry 2003;74(Suppl IV):iv13-iv17

r he provision of effective aids, orthoses, and environmental control systems for people with neurological disability is particularly challenging because of the spectrum of deficits that arise from disease or damage to the nervous system. Compare cardiovascular or musculoskeletal disease-the former often reduces exercise tolerance and mobility, the latter predominantly mobility, though in diseases such as rheumatoid arthritis also upper limb function. However, neurological disorders commonly compromise upper limb use (weakness, sensory loss, ataxia, apraxia, tremor), mobility, special senses of vision and hearing, and cognitive function. The basis of the disability thus may be complex. Rather than mobility reduced by angina or by joint stiffness and pain, mobility in neurological disease may be impaired through a combination of weakness, poor balance, proprioceptive loss, truncal ataxia, field cuts, diplopia on down gaze, and cognitive/perceptual problems.

\title{
AMELIORATING DISABILITY
}

There are several routes to ameliorating disability, and traditionally doctors have been involved in the rehabilitation process or specific techniques covered elsewhere. The subject of this paper is aids, orthoses, and environmental control systems.

- Aids: For the purpose of this review, aids can be defined as items designed to reduce disability. This is a relatively restrictive definition. By contrast, US legislation defines an aid as: "any item...whether acquired conventionally off the shelf, modified or customised, that is used to...improve functional capabilities of individuals with disabilities". This wider definition would embrace microwave ovens or TV remote controls.

- Orthoses: The Greek "orthos" means straight, normal or true. Orthoses are exoskeletal devices to limit or assist motion of some part of the body.

- Environmental control systems: These allow the disabled person to control aspects of their environment such as music, heating, light, ventilation or access.

The provision of aids, orthoses, and environmental control systems (hence referred to as aids) is not simply a matter of referring disabled patients for a social services occupational therapy (OT) assessment or to a physiotherapist. Aids are an integral part of the management of patients with complex disabilities and it is incumbent on the neurologist to understand the range available and the ways in which they can improve the independence of the patient. An understanding of the range of aids available and their potential provides the practising neurologist with broader perspectives of management. Consider your responses to the following queries:

- A young teacher with multiple sclerosis (MS), internuclear ophthalmoplegia, and nystagmus cannot work solely because of an inability to read due to nystagmus, which is much worse in one eye. You have tried oral medication and she declines botulinum toxin to ocular muscles. Can she be helped further?

- A young man with memory impairment and foot drop after head injury is seen for a medicolegal report. He complains he keeps forgetting appointments and forgets to use the diary the psychologist gave him. He has a splint from the physiotherapist but it keeps fracturing. Is there more that can be done?

- You lecture at a crowded patients' meeting. One of the audience wants to know if it will ever be possible to control a wheelchair by "brain power" alone. What do you answer?

It should be evident that neurologists regularly face clinical scenarios or questions which cannot appropriately be managed by referral to therapists. Neurologists need a basic understanding of aids, an awareness of certain specialist types, and a knowledge of how to refer. They will also benefit from monitoring developments in certain innovative areas which have the potential to alter patient care in the next few years.

Correspondence to: $\operatorname{Dr}$ C A Young, Walton Centre for Neurology \& Neurosurgery, Lower Lane, Liverpool, L9 7ப, UK; carolyn.young@ thewaltoncentre.nhs.uk

\section{ROLE OF NEUROLOGISTS}

Neurologists do not directly provide aids, orthoses, or environmental control systems. However they have several critical roles in appropriate equipment provision (table 1).

The foremost role must be expertly managing impairment to minimise disability, through a range of interventions including drugs, surgery, and specific rehabilitation techniques. 


\section{Table 1 Roles of neurologists in equipment provision}

1. Optimum treatment of impairment to minimise disability

2. Contribute to comprehensive assessment of disability

3. Referral, including where appropriate:

(a) Prognosis - are specified problems likely to change?

(b) Evolution-are other problems likely to develop from your

knowledge of the condition?

(c) Time course and life expectancy

4. Signing prescriptions recommended by others

5. Expertise and imagination
When referring patients for equipment provision to address residual disabilities, it is helpful to specify the less evident subtle impairments and disabilities. For example, when referring the patient with paraparesis and spasticity who has extensor spasms sometimes affecting the trunk and causing him to slide from the chair in spasm, mention the spasms as he may benefit from a ramped seat, a belt or a harness.

The neurologist has specific knowledge of the condition, whether the disabilities are static or anticipated to change, and provides invaluable information on likely future clinical course. Sharing this knowledge is particularly important for the less common conditions that the rehabilitation physician, therapist, or orthotist may be unfamiliar with. For example, two patients may be of comparable age and disability but the neurologist recognises that the outlook is very different if one has residual problems 10 years after acute disseminated encephalomyelitis (ADEM) and the other is entering the secondary progressive phase 10 years after diagnosis with MS.

Finally, the contribution neurologists may make to designing and improving new equipment should not be underestimated. Understanding the basis and nature of the problem to be solved, and the effect of the pathology on function, are key skills for being able to address these issues successfully.

\section{SOME AIDS FOR COMMON CLINICAL PROBLEMS}

The range of aids is vast and can be subdivided into different classes, only some of which will be relevant to neurological practice. Successful provision of many of them does not require any special knowledge on the part of the neurologist, simply an awareness of the clinical problem. Patients with leg length discrepancy, foot deformities or abnormal posture of foot or ankle may need specialised shoes. These are obtained by referral to orthotic clinics, often linked to orthopaedic services. Occupational therapists can advise on a very wide range of equipment to assist with activities of daily living. For outpatients the referral should normally go to the social services occupational therapist for that area.

Another source of provision is the charities-for example, the Red Cross will rent out wheelchairs, and Motor Neurone Disease Association local branches often loan equipment such as recliner chairs. Many walking aids are obtained through physiotherapy and may be provided following a clinical referral to improve gait or mobility. Physiotherapists may often be able to provide or prescribe orthoses, such as ankle foot orthoses (AFOs) or knee braces. The provision of splints may be through a specified commercial provider. It is always worth establishing whether the local rehabilitation medicine consultant offers an orthotics clinic for more difficult cases, so that medical expertise can be obtained.
In addition to these simple referrals there are several clinical problems where the neurologist may benefit from specialist information.

\section{Vision}

Patients who have functionally low vision should be referred to a low vision clinic and can benefit from page magnifiers, etc. Functionally impaired vision may be caused by neurological problems such as diplopia or nystagmus as much as reduced acuity. Orthoptists can assist stable diplopia with prisms but also teach patients to use simple aids to help adapt to hemianopia when carrying out tasks such as reading across the page.

The nystagmus troubled teacher in our first clinical vignette would benefit from occluding the eye with the severely oscillating image. Patches are not comfortable and are obtrusive to wear in public settings. She would benefit from a contact lens with the central area black, comfortably obscuring the image from that eye but cosmetically unnoticeable with the central opacity mimicking the pupil.

Specialised computer software is an increasingly useful aid. Screen settings for commercial programmes can be altered and in addition magnification programmes will expand images or part of the page or screen. Screen readers will convert the on-screen text to a synthesised voice or to Braille display with pins moving up and down to produce the Braille characters. Scanners can be used to import printed material to these programmes.

Patient with failing sight should be encouraged to learn to touch type. For those trying to navigate a keyboard using residual vision, it may be helpful to suggest a BigKeys board, with enlarged keys and characters enlarged 10 times.

Other common aids for those with poor vision include adapted phones and talking clocks/watches or tactile watches. In the kitchen, slicing knives with adjustable guards allow slices of variable thickness to be cut, and liquid level indicators make an audible signal when the liquid being poured into a vessel reaches a certain distance from the rim.

There are a wide variety of other aids for daily living and leisure for which the Royal National Institute for the Blind (RNIB) is a useful source of advice. One key area is talking books. These are available to anyone with near corrected visual acuity of N12 or less who takes out membership of the scheme.

\section{Mobility}

While patients obtain mobility aids such as sticks, tripods, zimmers, rollators, etc, through referral to physiotherapy, they can be referred specifically for wheelchairs to specialist wheelchair services. Standard chairs are provided at district level by therapists and rehabilitation engineers. Some more specialised assessment will be addressed at regional wheelchair clinics, often run by rehabilitation medicine physicians. This is particularly likely if a customised chair is needed-for example, if you are referring an individual with cerebral palsy and "windswept" trunk deformities necessitating moulded seating on a wheelchair base. The other more specialised area is powered chairs, either EPIC (electric powered indoor chairs) or EPIOC (electric powered indoor/outdoor chairs). National Health Service provision of these is restricted to people who cannot self propel and/or whose carers cannot push them. It is worth anticipating need as patients are distressed to lose independent mobility. For the person with motor neurone disease (MND), the timely referral for a 
powered wheelchair is as important as the timely referral for a percutaneous endoscopic gastrostomy (PEG) tube (see Leigh et al on p iv32).

Electric wheelchairs are usually controlled via a right or left hand joystick mounted in the arm of the chair. The settings on these can be damped for ataxic patients. Alternatively, chairs can be controlled by centrally mounted joysticks, chin/ head/foot controls, suck blow switches, eye movements, etc.

The control interface can be solved by technical ingenuity. Clinical problems such as poor vision, hemianopia, visual neglect, impaired cognition or impulsivity, are much more difficult to address. If these would make the powered wheelchair prescription unsafe such patients should not be referred.

\section{Falls}

Patients who are liable to falls should be referred for a personal alarm, usually worn as a pendant. Pressing this alerts one or two designated telephone numbers, usually relatives or neighbours or a paid emergency service. Physiotherapists can also teach individuals specific skills for getting up off the floor.

\section{Ataxia}

Aids for this difficult problem fall into three categories. The first is appendages like weighted wrist bands, intended to reduce the amplitude of the tremor and slow the speed and excursion of uncontrolled movements. The second is modifications of common items, designed to reduce the impact of incoordination, such as spouted beakers which will not spill. Thirdly, there are devices which allow the ataxic individual to perform a task through a novel route. An example of the latter is the so called Neater Eater, which is a mechanical feeding aid which damps tremor while transforming steady vertical hand motions into the scooping motion of a spoon.

\section{Communication}

This is a highly specialised area and rather than describe many different devices, continually changing as new technical possibilities arise, the neurologist should be grounded in some of the general principles. Communication is a vital skill, facilitating social interaction, and is fundamental for independence, autonomy, and quality of life.

Communication aids may be either standard or special. Standard aids are widely used and can readily be employed to aid communication with disabled people. Examples include gestures, diagrams, writing, drawing letters in the air or on the hand, even if just the first letter of the word is attempted. Special aids have been specifically developed for people with communication problems, such as sign language or communication aids. These special aids need prescription and/or training.

Within special communication aids there are two means of access, the choice of which depends on the individual's physical and cognitive capacities. Physical selection requires a physical movement to interact with the aid. Examples range from using a keyboard, pointing at an alphabet board, to using a laser pointer or pressing a switch.

Cognitive selection may be direct, such as typing on a conventional typewriter or pointing at a stylised image like "thirsty, want a drink". Scanning is slower and requires the user to choose when the correct option is reached. The aid may proceed at a set pace with the user choosing by a specialist switch. However, if the user freezes or has a spasm at the precise moment of choice, he/she needs to cycle through the whole repertoire again. It may be better to consider two switches, one which moves to the next option, and one which chooses. These methods are slow but easily learnt. Encoding demands that the person learns a new system, although working that system may be physically easy. Morse is the best known encoded system.

The output of the communication aid can be:

> sound-including speech

- visual display-ranging from slowly decoding a message spelled out on an alphabet board to reading an entire sentence on screen; some devices can print the screen display

- computer media-which in terms of ever shrinking hardware and acceptability and familiarity are likely to gain eminence.

\section{Driving}

Disabled individuals who are legally able may still have practical problems driving. Apart from standard modifications like automatics or power assisted steering, there are swivel seats, steering knobs, hand controls, or controls adapted for hemiplegic driving.

Patients can be referred to their local driving assessment centre where they will be evaluated, sometimes in a static car simulator or by a test drive. There is a statutory charge for such services.

\section{Memory}

The rehabilitation of memory mainly relies on psychological techniques. Aids can be useful, especially the simple ones requiring little training, such as diaries, memos, and cue cards. However, as our clinical vignette of the patient who complains he forgets to use his diary shows, using such aids is itself a memory task. External cueing systems such as the so called Neuropage have been shown in randomised controlled trials to be effective. The Neuropage is a radiopager which provides specific pre-programmed reminders of tasks on screen to individuals at designated times. The relative importance of the dual roles of mnemonic reminder and alerting device is uncertain. For those with intact memory but poor attention, a watch alarm may be sufficient.

\section{Others}

Computer technology is an expanding area which can be exploited to reduce disability. Referrals can be made to Ability Net or similar organisations. There is a wide range of aids for continence, which may be obtained through continence advisors. The needs of disabled children for aids for leisure and learning are a specialist area.

There are aids which are specific to particular diseases. The MND collar is useful for patients with weakness of neck extensors and seems both more effective and comfortable for people with MND than conventional collars (fig 1).

People with MND or muscular dystrophy may also need ventilatory support and/or suction devices. This aspect of care has been covered Howard and Davidson ${ }^{1}$ in an earlier article in this series.

Temperature sensitivity occurs with MS because partially demyelinated fibres show reduced conduction velocity with increasing temperature. Cooling jackets consist of a waistcoat with renewable ice packs and can be helpful in increasing work capacity for some temperature sensitive patients. 


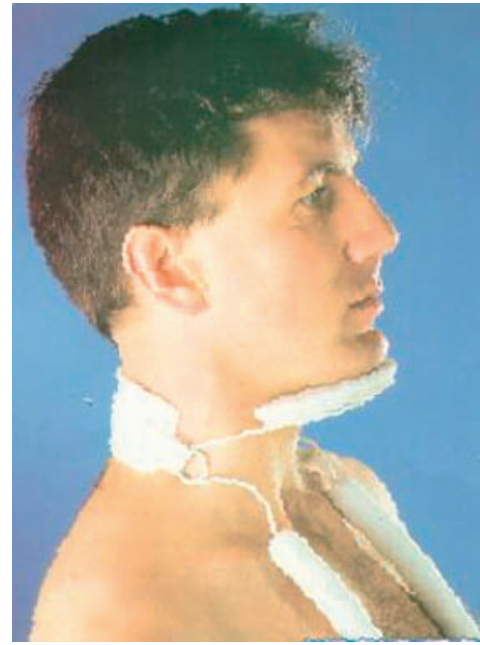

Figure 1 MND collar for individuals with motor neurone disease.

\section{ORTHOSES}

There is a very wide range or orthoses, some prescribed by therapists and some by orthotists and/or consultants in rehabilitation medicine. Orthoses can be static, without moving parts and designed to immobilise joints. Working orthoses allow the body to work in some functional way and often include a moving part, either free-moving or against resistance.

Most neurologists will be familiar with AFOs and knee orthoses. Hand orthoses are a specialist area with the aims of improving lost function, preserving hand function, and preventing contractures. Orthopaedic surgeons with a special interest in hand surgery usually have specialist physiotherapists linked to their teams.

Spinal orthoses include spinal braces and corsets, including devices designed to control juvenile scoliosis and kyphosis. The prescription of orthoses to assist upper limb function in patients with lesions of $\mathrm{C} 5$ or below is a specialist area which merits referral to the regional spinal injuries services.

Neurologists may contribute to developing better means of treating a given impairment than just a standard orthosis. Consider the common problem of dropped foot, where the toes are not lifted in the swing phase of walking. The standard orthosis is an AFO, such as was given to our hypothetical head injured patient in the clinical vignette. Decades ago Liberson and colleagues ${ }^{2}$ observed that electrical stimulation could aid "cases where the impulses coming from the central nervous system are lacking". They used a portable stimulator to drive the common peroneal nerve below the knee, timed by a pressure sensitive heel switch in the shoe on the affected side so that current flowed when the heel was lifted in swing phase and was interrupted in stance phase. The result was that the ankle was stimulated to dorsiflex in swing phase and gait improved. Since this early work there have been many improvements in stimulators and increasing knowledge of stimulation parameters. Many studies now confirm the benefits that can be gained from functional electrical stimulation to correct foot drop in upper motor neurone lesions. Stimulators for hand grasp in patients with C5/6 lesions are now also being increasingly used.

\section{ENVIRONMENTAL CONTROL SYSTEMS}

Enabling a disabled person to use a computer to control the immediate environment is a highly technical process.

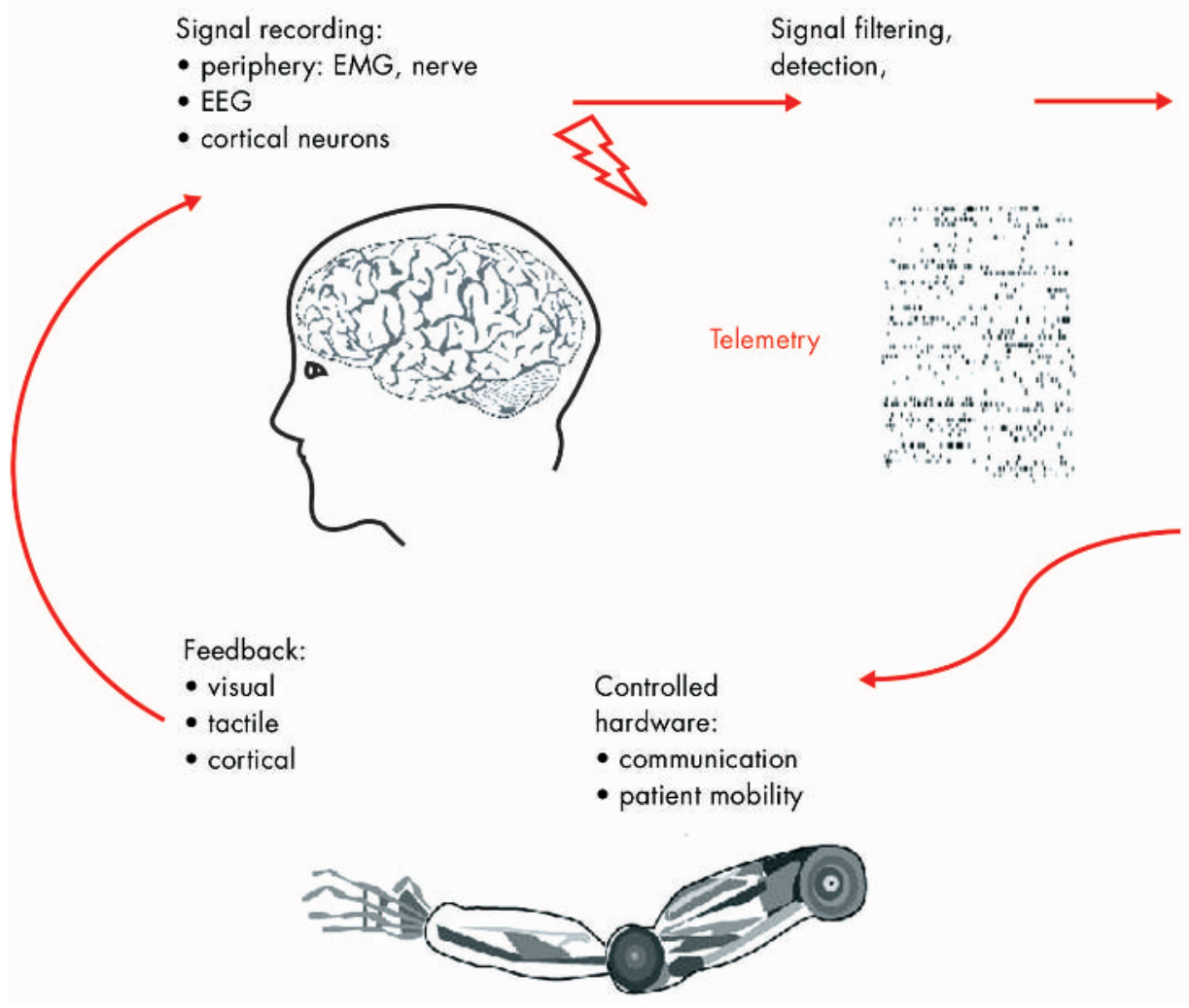

\section{Generation of control signals}
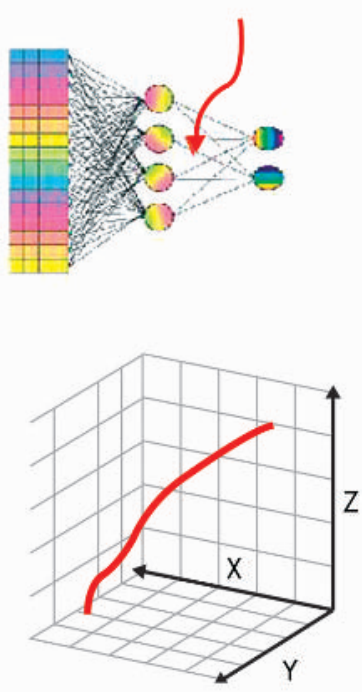

Figure 2 A potential interface with the brain. 
Rehabilitation medicine consultants or neurologists may take a special interest in medical assessment for environmental control systems and work with specialist rehabilitation electronic engineers to design appropriate systems. However, all neurologists should be aware of what is possible and how to refer.

Early systems provided nearly 50 years ago for survivors of the polio epidemics have dramatically improved with technological advances. Patients with severe physical disability can now use equipment like TVs, videos, doors, windows, lights, curtains, and motorised beds and chairs. Visitors at the front door can be seen on CCTV, talked to, and the door opened without an individual leaving their bed. An infra-red hands free phone allows the number to be dialled and a conversation to follow without the handset or phone being touched.

Computers and wheelchairs can be operated by a common environmental control system. User switches can be switches, joysticks or function via blink or eye movement. Both wired and wireless systems are possible.

\section{THE FUTURE}

Neurologists should monitor developments in aids, orthoses, and environmental control systems as this equipment can offer real benefits to the neurologically disabled patient. They may want to collaborate with exciting new developments relevant to their specialist knowledge and interest.

The use and applications of implanted neuroprostheses are expanding. Dorsal column stimulators, phrenic pacing, cochlear stimulators, and bladder controllers are widely used. Cerebellar stimulators for ataxia and upper limb stimulators are being tested. The use of lower limb stimulators for paraplegic standing and stepping is still largely at the research level. Visual cortex stimulators are being trialled, and there is also work on an artificial eye although the capacity to detect both static and moving objects and to replicate rods and cones is proving taxing.

Robots are being increasingly used both for rehabilitation due to their practice benefits and to address day to day problems of disability. The interface between user and machine is critical (fig 2).

Telecare may be useful for dementia, epilepsy or severe physical disability. "Smart homes" are houses where systems are activated automatically. They can perform falls, fire and water detection; temperature analysis; drug dispensing; and lifestyle monitoring. Lifestyle monitoring measures the interaction between user and environment in terms of mobility, use of cooking and washing facilities, sleep patterns, and toilet usage. While this may seem intrusive, trials suggest it is reassuring and acceptable to elderly people and that it may be extremely useful in enabling demented people to continue living safely in the community.

\section{CONCLUSIONS}

The start of this paper outlined three vignettes to illustrate the relevance of a knowledge of aids to the neurologist. The application and scope for aids, orthoses, and environmental control systems have been described, with some thought on future developments. However, open and innovative attitudes of neurologists and disabled people will be crucial to the deployment of new technology. How did you answer the third vignette of the question on whether brainpower could control wheelchairs in the future? This really asks whether an individual could interact with the environment through an alternative interface to bodily movement. The answer shows how the knowledge of the neurologist can influence more innovative designs for future equipment. Standard scalp EEG detects signals of $50-100 \mathrm{mV}$ in adults, quite sufficient to operate a switch. So thought power is already feasible, but only if we think.

\section{USEFUL WEBSITES}

Www.rnib.org.uk—visual impairment including talking books

www.dvla.gov.uk/at_a_glance/content.htm—driving regulations

Www.justmobility.co.uk/forum—driving assessment centres www.abilitynet.org.uk-computing

www.strath.ac.uk/Departments/NatCentre-orthoses

\section{ACKNOWLEDGEMENT}

I thank Dr Johan Wessberg and Nature for permission to reproduce fig 2 .

\section{REFERENCES}

1 Howard RS, Davidson C. Long term ventilation in neurogenic respiratory failure. J Neurol Neurosurg Psychiatry 2002;74(suppl III):iii24-30.

2 Liberson WT, Holmquest HI, Scott D, et al. Functional electrotherapy in stimulation of the peroneal nerve synchronised with the swing phase of gait of hemiplegic patients. Arch Phys Med Rehabil 1961;42:202-5. 